\title{
ERAPSO: Revealing the High Burden of Obesity in German Psoriasis Patients
}

Peter E. H. Schwarz · Andreas Pinter · Nima Melzer - Philipp Barteczek •

Maximilian Reinhardt

Received: May 22, 2019 / Published online: July 11, 2019

(C) The Author(s) 2019

\section{ABSTRACT}

Introduction: Plaque psoriasis is a chronic, systemic-inflammatory disease characterized by skin erythema, plaques and scaling, and is associated with different comorbidities like psoriatic arthritis, obesity, and cardiometabolic diseases. Obesity aggravates cardiovascular risk in psoriasis patients and can negatively affect psoriasis disease severity with proinflammatory adipocytokine production by adipocytes and infiltrated immune cells.

Methods: An online survey on nutrition and physical activity in psoriasis participants

Peter E. H. Schwarz and Andreas Pinter contributed equally.

Enhanced Digital Features To view enhanced digital features for this article go to https://doi.org/10.6084/ m9.figshare.8519390.

Electronic supplementary material The online version of this article (https://doi.org/10.1007/s13555019-0314-1) contains supplementary material, which is available to authorized users.

P. E. H. Schwarz

University Hospital Dresden, Dresden, Germany

A. Pinter

University Hospital Frankfurt, Frankfurt am Main, Germany

N. Melzer · P. Barteczek $(\bowtie) \cdot$ M. Reinhardt Novartis Pharma GmbH, Nuremberg, Germany e-mail: philipp.barteczek@novartis.com
(ERAPSO) collected cross-sectional data about eating behavior, physical activity, and prevalence of obesity and metabolic syndrome components from 9940 psoriasis participants in Germany.

Results: ERAPSO revealed a high burden of obesity in German psoriasis participants with $66.9 \%$ overweight or obese (body mass index $[\mathrm{BMI}] \geq 25 \mathrm{~kg} / \mathrm{m}^{2}$ ), compared to approximately $50 \%$ of the German general population. Affected body surface area (BSA), cardiovascular risk factors, and cardiovascular event frequency increased with increasing BMI. Severe psoriasis was more frequent in overweight participants and impaired engagement in weight loss diets and physical activity. Most German psoriasis participants $(90.2 \%)$ with $\mathrm{BMI} \geq 25 \mathrm{~kg} / \mathrm{m}^{2}$ perceived themselves as overweight. A minority $(21.2 \%)$ were currently exercising with the aim of losing weight, and $12.6 \%$ were currently on a weight loss diet. In overweight participants, just $13.3 \%$ stated that their physicians and/or health insurance offered specific weight loss programs. Conclusion: ERAPSO revealed inadequate medical care of obese psoriasis participants with insufficient support for weight loss through diet or increased physical activity. Although psoriasis participants showed an intact self-perception of obesity, they seemed to lack intrinsic motivation to lose weight, highlighting the need for external support in losing weight via tailored programs. Since psoriasis severity correlates with impairment in diets and sports, treating 
psoriasis adequately may allow participants to follow weight loss programs more successfully. Funding: Novartis Pharma GmbH, Nuremberg, DE.

Keywords: Cardiometabolic; ERAPSO; German; Obesity; Psoriasis; Secukinumab

\section{INTRODUCTION}

Psoriasis is an inflammatory disease typically characterized by formation of erythema, plaques, and scaling of the skin. Proinflammatory cytokines including TNF- $\alpha$, IL-23, or IL-17 are upregulated in psoriatic lesions and promote keratinocyte proliferation and inflammation in the skin $[1,2]$. These proinflammatory cytokines also promote systemic inflammation, which is associated with a range of comorbidity including increased cardiovascular risk [3]. This includes risk factors such as hyperlipidemia, hypertension, type 2 diabetes mellitus, obesity [4], and metabolic syndrome [5], affecting around $40 \%$ of psoriasis patients, compared to $13-27 \%$ of the general population $[6,7]$. Psoriasis patients are at increased risk of metabolic syndrome, which may be related to an increase in circulating adipocytokines $[5,8]$, mediating systemic proinflammatory effects [9].

The metabolic and cardiovascular comorbidity of psoriasis, such as obesity, is characterized by inflammatory processes, such as adipose tissue inflammation in obesity and insulin resistance or vascular inflammation in coronary artery disease [10-14]. A cycle of feedback between psoriasis and obesity is postulated, in which psoriasis causes systemic inflammation that in turn stimulates adipocytes to secrete proinflammatory cytokines that can promote cutaneous inflammation (Fig. 1) [15]. Obesity and metabolic syndrome negatively impact psoriasis disease severity, treatment response, and also aggravate the cardiovascular risk in psoriasis patients [9, 16-19]. Increased cutaneous inflammation is associated with vascular inflammation in psoriasis patients, which increases with psoriasis severity $[20,21]$.

A recent survey (PsoHealth) indicated that around $40 \%$ of German psoriasis patients are

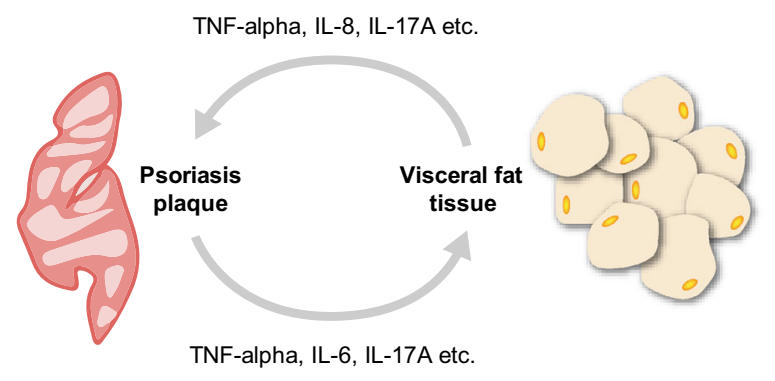

Fig. 1 Interaction of skin and adipose tissue in psoriasis and concomitant obesity. Psoriasis causes systemic inflammation that in turn stimulates visceral fat tissue to secrete proinflammatory cytokines that can promote cutaneous inflammation. TNF-alpha tumor necrosis factor alpha, IL interleukin

overweight, with an additional 30\% classified as obese [2]. According to the World Health Organization (WHO) 2008, overweight is considered as $\mathrm{BMI} \geq 25 \mathrm{~kg} / \mathrm{m}^{2}$, whereas obesity is considered as $B M I \geq 30 \mathrm{~kg} / \mathrm{m}^{2}$. To assess the relationship between psoriasis, obesity, patient behavior, and approach of care providers to weight management in individuals with psoriasis in the general population, a survey on health, nutrition, and physical activity was designed and conducted in German participants with self-reported psoriasis (hereafter referred to as participants) (Umfrage zu ERnährung und Aktivität bei PSOriasis-Patienten, or ERAPSO).

\section{METHODS}

\section{Study Objectives}

The study aim was to collect representative cross-sectional data from approximately 10,000 participants (comprising around $0.5 \%$ of individuals with psoriasis in Germany) about eating behavior, physical activity, prevalence of obesity and other factors contributing to metabolic syndrome, as well as the extent to which care providers offered support with weight management. The data was then used to assess the association between psoriasis, obesity, and patient behavior. A second aim was to give insight into current treatment practices and identify the potential need for intervention. 


\section{Survey Execution and Analysis}

ERAPSO was conducted with IT support from Clariness GmbH, Hamburg, Germany. The survey was online from 29 January to 21 March 2018. Completing the survey took approximately $10 \mathrm{~min}$. ERAPSO was promoted in German language on German websites, search engines, and social media, targeting individuals with psoriasis and encouraging their participation by asking for help in developing a better understanding of psoriasis.

According to the European consensus on definition of psoriasis severity, mild psoriasis is defined as $\mathrm{BSA} \leq 10 \%$ and moderate to severe disease is defined as BSA $>10 \%$ [22]. In this survey, BSA was self-assessed and entered online. To aid self-assessment of BSA, participants were shown a small graphic of a palm stating that the area of one palm is equal to $1.0 \%$ of the body surface.

For subgroup analyses, participants were divided into one group consisting of participants with mild disease (self-reported BSA $\leq 10 \%$ ) and another group consisting of participants with moderate to severe disease (selfreported BSA $>10 \%$ ). Participants reported data on (1) elevated cholesterol levels or other dyslipidemias, (2) impaired fasting glucose and/or diabetes mellitus, (3) arterial hypertension, and (4) waist circumference.

As a result of its observational and crosssectional nature, the data restricted itself to purely descriptive methodology, and no statistical testing was performed. Furthermore, as a result of this survey's self-reporting design, answers could not be confirmed.

\section{Compliance with Ethics Guidelines}

Ethics committee approval was not required because ERAPSO was conducted as an online survey. All the participants received explanations about the survey details. Informed consent was obtained from all participants for data storage, processing, and publication. This survey was performed in accordance with the Helsinki Declaration of 1964 and its later amendments.

\section{RESULTS}

\section{Demographic Data}

Overall, 15,693 individuals participated in this survey (Fig. 2). Of those, 97.0\% $(n=15,226)$ currently lived in Germany, and were included in the study. The study population comprised the $65.3 \%$ of the participants living in Germany who stated that they had been diagnosed with psoriasis $(n=9940$, "true completers"), the $32.3 \%$ of participants who had never been diagnosed with psoriasis $(n=4923)$, and $2.4 \%$ who were not sure $(n=363)$, and were therefore excluded from the analysis. All of the following results describe only participants living in Germany with a confirmed diagnosis of psoriasis $(n=9940)$.

Within the study population, $55.1 \%$ $(n=5477)$ were female and $44.9 \%(n=4463)$ were male, with a mean age at survey completion of $51.3 \pm 14.0$ years (Table 1 ). A majority $(65.1 \% ; n=6474)$ were currently being treated for psoriasis by their dermatologist and $14.5 \%$ $(n=1439)$ were being treated by other physicians, for example general practitioners. A minority of participants $(3.7 \% ; n=368)$ consulted a homeopath or alternative practitioner (multiple selections were possible). Nearly onequarter of participants $(25.3 \% ; n=2518)$ stated

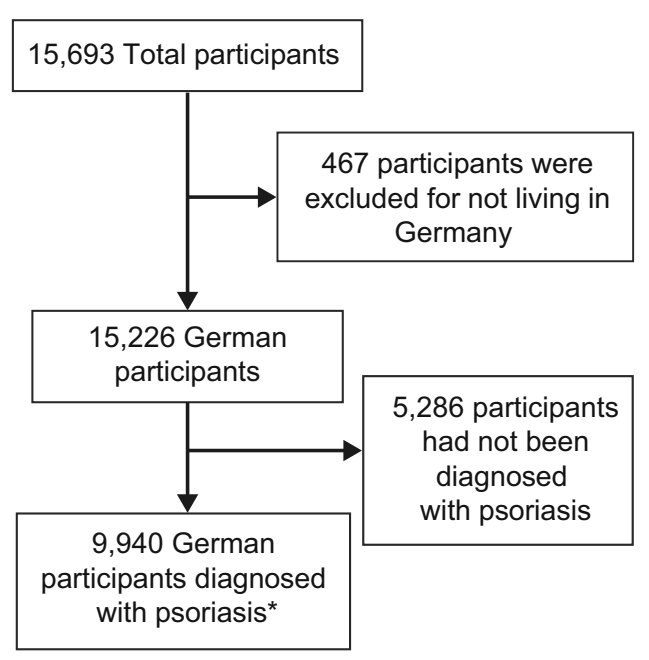

*Only these participants are described in this manuscript, 'true completers'

Fig. 2 Flow chart of ERAPSO survey participants 
Table 1 Baseline characteristics

\begin{tabular}{ll}
\hline Characteristic & $\boldsymbol{N}=\mathbf{9 9 4 0}$ \\
\hline Age (years) & $51.3 \pm 14.0$ \\
Male gender & $4463(44.9 \%)$ \\
Body surface area (\%) & $12.7 \pm 19.1$ \\
Mild psoriasis & $7404(74.5 \%)$ \\
Moderate to severe psoriasis & $2536(25.5 \%)$ \\
Time since diagnosis (years) & $23.0 \pm 16.0$ \\
Currently treated for psoriasis & $7422(83.3 \%)$ \\
Previous systemic treatment & $4222(42.5 \%)$ \\
Weight $(\mathrm{kg})$ & $83.8 \pm 21.2$ \\
BMI (kg/m ${ }^{2}$ ) & $28.5 \pm 14.1$ \\
Waist circumference (male, cm) & $92.7 \pm 17.0$ \\
\hline
\end{tabular}

Of 15,693 total participants, 9940 currently live in Germany, have self-reported a diagnosis of psoriasis, and are included in this study. Mild psoriasis is defined as body surface area $(\mathrm{BSA}) \leq 10 \%$ and moderate to severe disease is defined as $\mathrm{BSA}>10 \%$

$B M I$ body mass index

that they did not receive any treatment for psoriasis at all.

\section{Psoriasis Severity}

In this survey, the mean BSA affected by psoriasis was $12.7 \pm 19.1 \%$ (Table 1 ). The majority of participants $(74.5 \% ; n=7404)$ had mild disease while $25.5 \%(n=2536)$ were affected by moderate to severe psoriasis. Participants with mild psoriasis had a mean self-reported BSA of $4.0 \pm 2.9 \%$ while participants with moderate to severe psoriasis had a mean self-reported BSA of $38.0 \pm 23.5 \%$. This is consistent with established prevalence of moderate to severe psoriasis.

\section{Obesity}

Mean participant body weight was $83.8 \pm 21.2 \mathrm{~kg}$. Mean BMI was $28.5 \pm 14.1 \mathrm{~kg} /$ $\mathrm{m}^{2}$ (Table 1). Figure 3 shows the distribution of

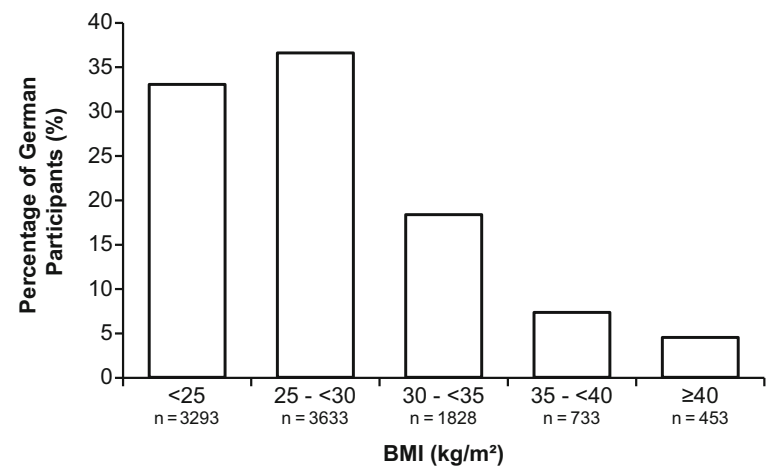

Fig. 3 BMI distribution shows high burden of obesity in German participants. $66.9 \%$ of German participants are overweight or obese $\left(\mathrm{BMI} \geq 25 \mathrm{~kg} / \mathrm{m}^{2}\right)$. In comparison, approximately $50-60 \%$ of the total German population is overweight or obese [23]. Total $N=9940$. BMI body mass index

BMI in the overall study population. In this population, $66.9 \%(n=6647)$ of all participants with psoriasis were overweight or obese (defined by the WHO 2008 as $\mathrm{BMI} \geq 25 \mathrm{~kg} / \mathrm{m}^{2}$ ). In comparison, approximately $50-60 \%$ of the general German population is overweight or obese [23]. Most participants (90.2\%) with $\mathrm{BMI} \geq 25 \mathrm{~kg} / \mathrm{m}^{2}$ perceived themselves as being overweight. Figure 4 shows the association of BMI with psoriasis severity. Participants with high BMI tended to show increased reported BSA.

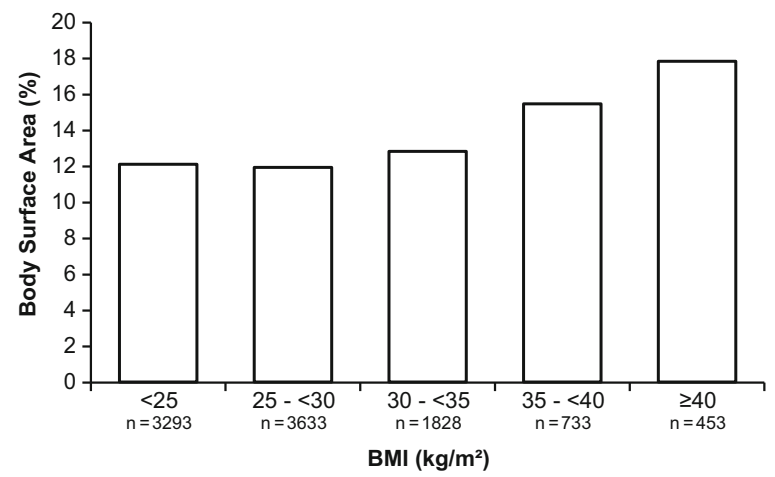

Fig. 4 Affected body surface area increases with BMI in ERAPSO participants. BMI subgroups are shown with associated mean affected body surface area. Total $N=9940, n=$ number of participants per BMI subgroup. BMI body mass index 


\section{Cardiovascular Profile}

Within the study population, 5.9\% $(n=582)$ had reported coronary heart disease, $2.5 \%$ $(n=246)$ reported angina pectoris, $3.3 \%$ $(n=331)$ had signs of myocardial infarction, and $2.5 \% \quad(n=251)$ reported having had a stroke. On division by BMI subgroups, higher BMI was associated with increased prevalence of cardiovascular risk factors (CVRFs) and of cardiovascular events (CVEs) (Figs.S1 and S2, electronic supplementary material).

Of all the participants $(n=9940), 23.1 \%$ $(n=2297)$ reported high cholesterol or other dyslipidemias. Impaired fasting glucose was reported by $9.0 \%(n=894)$ of participants and 9.5\% $(n=940)$ had been diagnosed with diabetes mellitus. In total $34.4 \%(n=3422)$ were diagnosed with arterial hypertension. Mean waist circumference in women was $88.4+16.5 \mathrm{~cm}$ and that in men was $92.7+17.0 \mathrm{~cm}$. More women had elevated waist circumference $(n=3961 / 5477$ [72.3\%] above $78.7 \mathrm{~cm})$ than men $(n=855 / 4463$ [19.2\%] above $100.0 \mathrm{~cm})$. These are common cutoff values for elevated waist circumference [24].

\section{Diet and Physical Activity}

Among overweight participants, $12.6 \%$ $(n=836)$ were currently on a weight loss diet. The majority of overweight participants had tried weight loss diets already $(60.7 \%, n=4038)$ and $55.6 \% \quad(n=2244)$ had been successful (completed to participants' satisfaction) with weight loss diets at least once.

Food was said to influence psoriasis severity by $25.9 \%(n=1914)$ of participants with mild psoriasis and $31.0 \%(n=785)$ of participants with moderate to severe psoriasis. Participants who stated that food can have positive or negative effects on their disease severity were asked to evaluate different types of food and how it affects their disease (Fig. 5). While vegetables, fish, potatoes, pasta, rice, and dairy products were perceived to have positive effects on psoriasis severity in many participants, convenience products, fast food, sweets, and alcohol

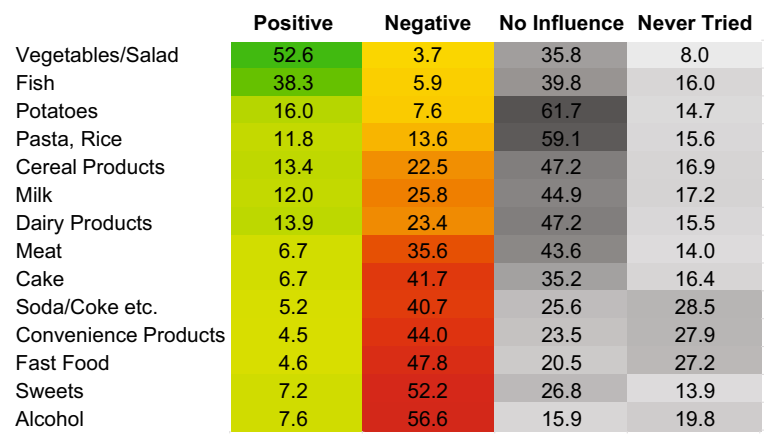

Fig. 5 Positive and negative effects of different types of food on psoriasis. All data shown are the percentage of participants. Participants $(n=2699$ [27.2\%] of the total population) stated that food can have positive or negative effects on their disease severity. They were asked to evaluate different types of food and how it affects their disease. Darker color shades represent a higher percentage

appeared to have negative effects on psoriasis severity in approximately $50 \%$ of participants in the overall population. Within the ERAPSO population, $9.0 \%(n=346)$ of participants with mild psoriasis say that psoriasis has prevented them from losing weight by diet, whereas $20.3 \%$ $(n=257)$ of participants with moderate to severe psoriasis say that their psoriasis has prevented them from losing weight by diet.

Only $21.2 \%(n=1408)$ of overweight participants reported currently exercising to lose weight. Participants who were overweight reported greater discomfort with physical activity than those with $\mathrm{BMI}<25 \mathrm{~kg} / \mathrm{m}^{2}$ (Fig. 6a) when their psoriasis is active. Onequarter $(25.6 \% ; n=904)$ of participants with mild psoriasis and $46.6 \%(n=514)$ of participants with moderate to severe psoriasis said that psoriasis has prevented them from exercising at least once. Figure $6 \mathrm{~b}$ illustrates how active psoriasis is associated with discomfort with physical activities in participants with increasing disease severity, to the extent of causing total abandonment of physical activity. Eighteen percent of participants with mild psoriasis and $25.8 \%$ of participants with moderate to severe psoriasis said that exercise can influence their disease severity. These participants were asked to evaluate different types of physical activity and how they affected their disease severity (Fig. 7). While walking, cycling, and endurance 


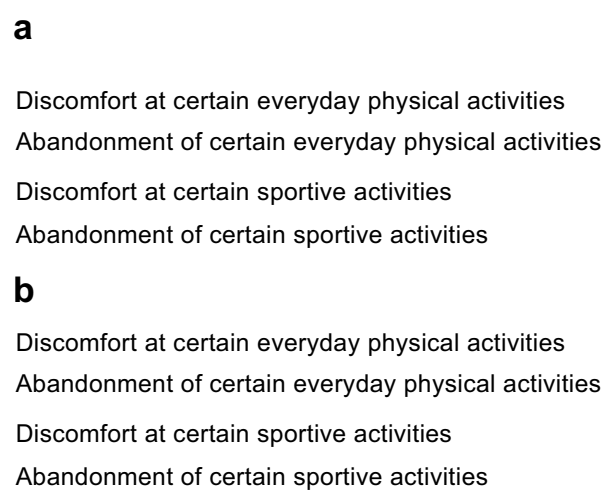

Fig. 6 Active psoriasis impairs physical exercise especially in overweight participants (a) and participants with moderate to severe psoriasis (b). All data shown are the percentage of participants. Participants were asked how active psoriasis disease affects their physical activities. The figure shows the percentage of participants suffering from impairment of physical activities. Impairment of sportive activities was examined in a population doing sports with

\begin{tabular}{lcccc} 
& Positive & Negative & No Influence & Never Tried \\
\hline Walking & 41.0 & 17.4 & 39.5 & 2.1 \\
Cycling & 32.4 & 16.5 & 38.1 & 13.0 \\
Endurance Sports & 26.6 & 19.8 & 30.1 & 23.5 \\
Stretching & 27.6 & 14.5 & 38.8 & 19.2 \\
Stair Climbing & 23.0 & 25.2 & 49.4 & 2.5 \\
Weight Training & 13.1 & 21.6 & 29.1 & 36.1 \\
Housework & 19.4 & 31.1 & 46.7 & 2.8
\end{tabular}

Fig. 7 Different types of physical exercise can have positive and negative effects on psoriasis. All data shown are the percentage of participants. Participants $(n=1989$ [20.0\%] of the total population) stated that physical activity can have positive or negative effects on their disease severity. They were asked to evaluate different types of physical activities and how they affected their disease. Darker color shades represent a higher percentage

sports showed positive effects in $41.0 \%, 32.4 \%$, and $26.6 \%$ of participants, respectively, housework showed a negative impact on psoriasis severity in $31.1 \%$ of participants.

Nearly one-third $(35.7 \% ; n=2374)$ of overweight participants have been approached by their treating physician to lose weight by going on a diet or exercising. Only $13.3 \%(n=887)$ of overweight participants stated that their physicians and health insurances offer specific programs to lose weight using diets or physical activity.

\begin{tabular}{|c|c|c|}
\hline $\begin{array}{c}\text { BMI } \\
<25 \mathrm{~kg} / \mathrm{m}^{2}\end{array}$ & $\begin{array}{c}\text { BMI } \\
\geq 25 \mathrm{~kg} / \mathrm{m}^{2}\end{array}$ & \multirow{3}{*}{$\begin{array}{l}\text { Total Population } \\
\mathrm{N}=9940\end{array}$} \\
\hline 29.9 & 39.7 & \\
\hline 19.9 & 26.1 & \\
\hline 27.3 & 37.2 & \multirow{3}{*}{$\begin{array}{l}\text { Population doing sports } \\
\mathrm{N}=7717\end{array}$} \\
\hline 23.9 & 31.5 & \\
\hline$B S A \leq 10 \%$ & $B S A>10 \%$ & \\
\hline 31.9 & 50.0 & \multirow{2}{*}{$\begin{array}{l}\text { Total Population } \\
\mathrm{N}=9940\end{array}$} \\
\hline 20.0 & 35.7 & \\
\hline 29.6 & 46.8 & \multirow{2}{*}{$\begin{array}{l}\text { Population doing sports } \\
\mathrm{N}=7717\end{array}$} \\
\hline 24.9 & 41.1 & \\
\hline
\end{tabular}

varying intensity. a Illustrates impairment of physical activities in participants with normal weight $\left(\right.$ BMI $\left.<25 \mathrm{~kg} / \mathrm{m}^{2}\right)$ compared to overweight participants $\left(B M I \geq 25 \mathrm{~kg} / \mathrm{m}^{2}\right)$. b Reports the same outcomes dependent on mild psoriasis (BSA $\leq 10 \%)$ or moderate to severe psoriasis (BSA $>10 \%)$. Darker color shades represent a higher percentage. BMI body mass index, BSA body surface area

\section{DISCUSSION}

ERAPSO identified a higher prevalence of overweight and obesity among participants than in the general German population, where around $50-60 \%$ are overweight or obese [23]. Rates of overweight and obesity in participants matched those determined in PsoHealth [2]. There is not only an objective burden of obesity but also a subjectively perceived burden of obesity, as participants show intact self-perception regarding overweight/obesity. Despite this, only a minority of overweight participants are currently attempting to lose weight by diet or exercise. This underlines the need for professional external support in multiple approaches.

Awareness of obesity or the necessity to treat obesity in psoriasis patients appears relatively low among physicians, as only a minority of participants were offered weight loss programs. Awareness of the negative impact of obesity and metabolic syndrome on psoriasis disease severity needs to be raised, and the efficacy of treatment options established. For example, weight loss combined with cyclosporine therapy improves psoriasis more than cyclosporine alone [25]. The combined efficacy of weight loss and secukinumab treatment for plaque psoriasis 
is under current investigation (METABOLYX trial, NCT03440736).

Compared to ERAPSO findings on the prevalence of CVRFs in participants, in Germany $28.1 \%$ of men and $27.1 \%$ of women overall have dyslipidemia, with a significant increase in prevalence with increasing age and with no significant differences between sexes [26]. Prevalence of arterial hypertension is around $40-50 \%$ in adults in Germany (Robert Koch Institut, Statistisches Bundesamt 2018). Type 2 diabetes occurs in $7-8 \%$ of the general German population (Deutsche Diabetes Gesellschaft 2018). ERAPSO participants showed a similar prevalence of dyslipidemia, arterial hypertension, and diabetes mellitus to the general German population. Increasing prevalence of CVRFs with growing BMI translated into increased incidence of CVEs.

Participants with moderate to severe psoriasis perceived stronger positive and negative influences of food and sports on their disease than participants with mild psoriasis. Psoriasis severity was identified by participants as a limiting factor for engaging in exercise, as might be expected given physical symptoms and concerns regarding outer appearance in sports clothing. Interestingly, some participants reported that severity of psoriasis also impacted their capacity to follow a specific diet. This suggests an intersection between the psychological effects of psoriasis, including depression and, potentially, emotional eating, and obesity, and may warrant further research. In addition, treating psoriasis and adequate control of skin symptoms could help participants to lose weight since psoriasis severity is associated with impairment in diets and exercise. Participants reported "healthy" foods such as vegetables having a positive effect on their psoriasis, whereas processed food, sweets, and alcohol were more frequently labelled as having negative effects. A perceptual effect cannot be ruled out, as for instance it is unclear whether increased alcohol consumption in psoriasis participants is a risk factor for the disease [27]. However, when giving advice on nutrition and diets for psoriasis patients, physicians can continue to recommend "healthy" food like fiberrich vegetables and fish, which seems to have positive effects on participants' perception of disease. In addition, when giving advice on physical exercise, physicians can continue to recommend daily physical activities like endurance sports, cycling, or walking. Furthermore, endurance sports, cycling, or walking are often more practical than resistance training to include as daily activities and they have a higher chance to burn body fat compared to resistance training. ERAPSO revealed participants' perceptions of weight loss treatment as being limited. In terms of weight management, diet, and exercise, treatment offerings appeared to be insufficient.

Limitations of ERAPSO include the self-selecting patient population. For example, severely depressed participants may be less likely to complete online surveys. A further limitation is that all data collected, including psoriasis diagnosis, were based on participants self-reporting of their health information, not on physician's records. Finally, differences in digital literacy between participants compared with the general population might be a further source of bias, with the ERAPSO participants being a self-selecting digitally literate subpopulation.

\section{CONCLUSIONS}

ERAPSO showed a high prevalence of overweight and obesity among participants in Germany, with psoriasis severity being associated with BMI. A relationship between obesity and $\mathrm{CV}$ risk factors or events was also apparent. Only a minority of overweight/obese participants were currently on a diet or exercising to lose weight, and few were approached by their physician with weight loss recommendations, indicating a lack of professional weight loss support for psoriasis patients. Psoriasis severity was associated with impairment in participation and success of diets and physical activity; therefore, optimal psoriasis disease control could in itself be a means to facilitate patients' weight loss attempts. ERAPSO shows an unmet medical need in obese individuals with self-reported psoriasis, with insufficient offerings for professional support to lose weight or increase physical activity. 


\section{ACKNOWLEDGEMENTS}

We thank the participants of the study.

Funding Sources. This research and article processing charges were funded by Novartis Pharma GmbH, Nuremberg, DE. The analyses presented here were funded by Novartis Pharma GmbH, Germany. All authors had full access to all of the data in this study and take complete responsibility for the integrity of the data and accuracy of the data analysis.

Medical Writing and/or Editorial Assistance. Medical writing assistance was provided by Evelyn Altemeyer and Jackie L. Johnson, Ph.D., Novartis Ireland Ltd, and funded by Novartis Pharma GmbH, Germany in line with GPP3.

Authorship. All named authors meet the International Committee of Medical Journal Editors (ICMJE) criteria for authorship for this article, take responsibility for the integrity of the work as a whole, and have given their approval for this version to be published.

Disclosures. Peter E. H. Schwarz has Grant/ Research Support from Deutsches Zentrum für Diabetesforschung (DZD), European Commission, EU Directorate Public Health, BMBF; has served on the Speaker's Bureau for the European Commission, AOKplus, Ascensia, Novartis, Lifescan, MERCK, Novo, Lilly, MSD, Boehringer, REHASAN, Sanofi; is a Consultant for AOKplus, Ascensia, TUMAINI Institut für Präventionsmanagement, REHASAN and is affiliated with TUMAINI Institut für Präventionsmanagement. Andreas Pinter has worked as an investigator and/or speaker and/or advisor for following pharmaceutical companies: AbbVie, AlmirallHermal, Amgen, Biogen Idec, Boehringer-Ingelheim, Celgene, GSK, Eli-Lilly, Galderma, Hexal, Janssen, LEO-Pharma, Medac, Merck Serono, Mitsubishi, MSD, Novartis, Pascoe, Pfizer, Tigercat Pharma, Regeneron, Roche, Sandoz Biopharmaceuticals, Schering-Plough, and UCB Pharma. Nima Melzer is an employee of Novartis Pharma GmbH. Philipp Barteczek is an employee of Novartis Pharma GmbH.
Maximilian Reinhardt is an employee of Novartis Pharma GmbH.

Compliance with Ethics Guidelines. Ethics committee approval was not required because ERAPSO was conducted as an online survey. All the participants received explanations about the survey details. Informed consent was obtained from all participants for data storage, processing, and publication. This survey was performed in accordance with the Helsinki Declaration of 1964 and its later amendments.

Data Availability. Novartis is committed to sharing with qualified external researchers, access to patient-level data, and supporting clinical documents from eligible studies. These requests are reviewed and approved by an independent review panel on the basis of scientific merit. All data provided is anonymized to respect the privacy of patients who have participated in the survey in line with applicable laws and regulations.

Open Access. This article is distributed under the terms of the Creative Commons Attribution-NonCommercial 4.0 International License (http://creativecommons.org/licenses/ by-nc/4.0/), which permits any noncommercial use, distribution, and reproduction in any medium, provided you give appropriate credit to the original author(s) and the source, provide a link to the Creative Commons license, and indicate if changes were made.

\section{REFERENCES}

1. Chiricozzi A, Romanelli P, Volpe E, Borsellino G, Romanelli M. Scanning the immunopathogenesis of psoriasis. Int J Mol Sci. 2018;19(1):179.

2. Jacobi A, Langenbruch A, Purwins S, Augustin M, Radtke MA. Prevalence of obesity in patients with psoriasis: results of the National Study PsoHealth3. Dermatology. 2015;231(3):231-8.

3. Griffiths CEM, Jo S-J, Naldi L, et al. A multidimensional assessment of the burden of psoriasis: results from a multinational dermatologist and patient survey. Br J Dermatol. 2018;179(1):173-81. 
4. Shah K, Mellars L, Changolkar A, Feldman SR. Realworld burden of comorbidities in US patients with psoriasis. J Am Acad Dermatol. 2017;77(2): 287-92.e4.

5. Takahashi $H$, Iizuka $H$. Psoriasis and metabolic syndrome. J Dermatol. 2012;39(3):212-8.

6. Love TJ, Qureshi AA, Karlson EW, Gelfand JM, Choi HK. Prevalence of the metabolic syndrome in psoriasis: results from the National Health and Nutrition Examination Survey, 2003-2006. Arch Dermatol. 2011;147(4):419-24.

7. Neuhauser H, Ellert U. Estimation of the metabolic syndrome prevalence in the general population in Germany. J Public Health. 2008;16(3):221-7.

8. Danielsen K, Wilsgaard T, Olsen AO, et al. Elevated odds of metabolic syndrome in psoriasis: a population-based study of age and sex differences. $\mathrm{Br} \mathrm{J}$ Dermatol. 2015;172(2):419-27.

9. Carrascosa JM, Rocamora V, Fernandez-Torres RM, et al. Obesity and psoriasis: inflammatory nature of obesity, relationship between psoriasis and obesity, and therapeutic implications. Actas Dermosifiliogr. 2014;105(1):31-44.

10. Bluher M. Adipose tissue inflammation: a cause or consequence of obesity-related insulin resistance? Clin Sci. 2016;130(18):1603-14.

11. Ali M, Girgis S, Hassan A, Rudick S, Becker RC. Inflammation and coronary artery disease: from pathophysiology to Canakinumab Anti-Inflammatory Thrombosis Outcomes Study (CANTOS). Coron Artery Dis. 2018;29(5):429-37.

12. Shoelson SE, Goldfine AB. Getting away from glucose: fanning the flames of obesity-induced inflammation. Nat Med. 2009;15(4):373-4.

13. Prussick RB, Miele L. Nonalcoholic fatty liver disease in patients with psoriasis: a consequence of systemic inflammatory burden? $\mathrm{Br} \mathrm{J}$ Dermatol. 2018;179(1):16-29.

14. $\mathrm{Xu} \mathrm{X}, \mathrm{Su} \mathrm{L}, \mathrm{Gao} \mathrm{Y}$, Ding Y. The prevalence of nonalcoholic fatty liver disease and related metabolic comorbidities was associated with age at onset of moderate to severe plaque psoriasis: a cross-sectional study. PLoS One. 2017;12(1):e0169952.

15. Reich K. The concept of psoriasis as a systemic inflammation: implications for disease management. J Eur Acad Dermatol Venereol. 2012;26(Suppl 2):3-11.

16. Versini M, Jeandel PY, Rosenthal E, Shoenfeld Y. Obesity in autoimmune diseases: not a passive bystander. Autoimmun Rev. 2014;13(9):981-1000.
17. Yanaba K, Umezawa $Y$, Ito T, et al. Impact of obesity on the efficacy of ustekinumab in Japanese patients with psoriasis: a retrospective cohort study of 111 patients. Arch Dermatol Res. 2014;306(10):921-5.

18. Gelfand JM, Neimann AL, Shin DB, Wang X, Margolis DJ, Troxel AB. Risk of myocardial infarction in patients with psoriasis. JAMA. 2006;296(14):1735-41.

19. $\mathrm{Hu} \mathrm{SC}$, Lan CE. Psoriasis and cardiovascular comorbidities: focusing on severe vascular events, cardiovascular risk factors and implications for treatment. Int J Mol Sci. 2017;18(10):2211.

20. Gistera A, Hansson GK. The immunology of atherosclerosis. Nat Rev Nephrol. 2017;13(6): 368-80.

21. Jamasbi J, Ayabe K, Goto S, Nieswandt B, Peter K, Siess W. Platelet receptors as therapeutic targets: past, present and future. Thromb Haemost. 2017;117(7):1249-57.

22. Mrowietz U, Kragballe K, Reich K, et al. Definition of treatment goals for moderate to severe psoriasis: a European consensus. Arch Dermatol Res. 2011;303(1):1-10.

23. AOK-Bundesverband (DE). AOK-Familienstudie [internet]. Berlin: AOK-Bundesverband; 2018 [cited 2018 May 10]. Available from: https://www.aok.de/ pk/fileadmin/user_upload/Universell/05-ContentPDF/aok-familienstudie-2018.pdf.

24. Alberti KG, Eckel RH, Grundy SM, et al. Harmonizing the metabolic syndrome: a joint interim statement of the International Diabetes Federation Task Force on Epidemiology and Prevention; National Heart, Lung, and Blood Institute; American Heart Association; World Heart Federation; International Atherosclerosis Society; and International Association for the Study of Obesity. Circulation. 2009;120(16):1640-5.

25. Gisondi P, Del Giglio M, Di Francesco V, Zamboni $\mathrm{M}$, Girolomoni G. Weight loss improves the response of obese patients with moderate-to-severe plaque psoriasis to low-dose cyclosporine therapy: a randomized, controlled, investigator-blinded clinical trial. Am J Clin Nutr. 2008;88:1242-7.

26. Scheidt-Nave C, Du Y, Knopf H, et al. Prevalence of dyslipidemia among adults in Germany: results of the German health interview and examination survey for adults (DEGS 1). Bundesgesundheitsblatt Gesundheitsforschung Gesundheitsschutz. 2013;56(5-6):661-7.

27. Brenaut E, Horreau C, Pouplard C, et al. Alcohol consumption and psoriasis: a systematic literature review. J Eur Acad Dermatol Venereol. 2013; 27(Suppl 3):30-5. 